\title{
Evaluation of results of Total Elbow Arthroplasty with a Linked Semiconstrained Prosthesis
}

\author{
Saran $\mathbf{R}^{1}$, Saxena $\mathbf{A}^{2}$ \\ ${ }^{1}$ Dr Rajat Saran, Associate Professor, Department of Orthopaedics, Chirayu Medical College, Bhopal. ${ }^{2}$ Dr Anand Saxena, \\ Associate Professor, Department of Surgery, L N Medical College, Bhopal, MP, India
}

Address for correspondence: Dr Rajat Saran, Email: saran.rajat@gmail.com

\begin{abstract}
Introduction: Extremity arthroplasties have been performed for 150 to 200 years, but no arthroplasty has been developed that universally meets the needs of every patient with a disabled elbow joint. Although the frequency of total elbow arthroplasty is much less as compared to hip and knee, it has definitely made an emphatic place for itself in the world of joint replacements. Methods: This is a study of nine patients out of eleven, as two patients were lost to follow up very early in the process. Between August 2004 to August 2014 nine patients were taken up for total elbow replacement for different indications which included neglected supracondylar fractures of the humerus sustained in childhood, rheumatoid arthritis and grossly comminuted fractures, closed and compound, of the distal humerus. Results: Out of the nine patients considered, seven had no pain whereas two patients with a comminuted compound fracture of the lower end of humerus, with one patient in whom myositis was excised, complained of regular pain postoperatively. Conclusion: The aim of this prospective study is to evaluate the results of the linked prosthesis in total elbow arthroplasty for different indications.
\end{abstract}

Keywords: Total elbow arthroplasty, linked prosthesis, Semiconstrained Prosthesis

\section{Introduction}

History suggests that non operative treatment was usually suggested for gross traumatic pathologies of the distal humerus. In 1969 Riseborough and Radin [1] opined that operative treatment was usually associated with poor outcomes and was unpredictable for comminuted intra articular distal humerus fractures and recommended conservative treatment.

Brown and Morgan [2] also claimed satisfactory results with early active motion conservatively. Over the last 25 years the Arbeitsgemeinschaft fur Osteosynthesefragen (AO) principles of anatomic articular reduction and rigid internal fixation to allow early post operative motion have however reported good results [3-10]. In younger patients this mode of treatment has come to stay as the gold standard.

Total elbow arthroplasty as a primary treatment has been suggested as an indication in elderly patients because of the poor bone quality which poses problems in restoring anatomical reduction and affording rigid

Manuscript received: $16^{\text {st }}$ Aug 2014

Reviewed: $29^{\text {th }}$ Aug 2014

Author Corrected: $16^{\text {th }}$ Sep 2014

Accepted for Publication: $25^{\text {th }}$ Sep 2014 internal fixation [11]. As compared to other joints, elbow being a small joint its stability mainly depends upon the structure and strength of the ligaments. Although it was initially used in arthropathies caused by rheumatoid arthritis, indications were extended also to other problems which led to increased demands on the implants increasing their failures[12] Rheumatoid arthritis causing inflammatory arthropathy is an absolute indication for elbow arthroplasty. Posttraumatic osteoarthritis, acute distal humerus fractures, non unions and reconstruction after tumour resection surgery may also be included as relatively valid indications. Elbow arthroplasty is very successful in terms of pain relief, motion and function. However, its complication rate remains higher than arthroplasties of other joints.

\section{Materials and Methods}

By and large, two designs of implants are in vogue, which have different mechanisms of linking the ulnar and humeral components. The goal is to avoid subluxation or dislocation episodes of the elbow after surgery. Linked implants used earlier were constrained hinges which allowed only flexion and extention transmitting high 
stresses to the interface between the implant and the cement, resulting in failures. The linking mechanism on the other hand in semi constrained implants affords a sloppy hinge with an element of rotation and varusvalgus play. This limited transmission of the stress to the interface with some improvisations in the design has prolonged the viability of the prosthesis.

From August 2004 to February 2013, eleven cases were operated upon for total elbow arthroplasty for different indications. Out of these eleven cases, two were lost to follow up after three and eight weeks respectively and hence were not included in this study. The maximum follow up of the nine patients in this study has been for eight and a half years and the minimum has been for five months. The age of the patients ranged from 23 years to 62 years which included six males and three females. The indications for which the patients were included in this study were namely neglected supracondylar fractures of the humerus, rheumatoid arthritis and badly comminuted fractures of the lower end of humerus (bag of bones). Two males aged 23 and 32 years had flail, weak and wasted elbows with no flexion or extension at the proper elbow joint. However, the forearm was dangling from a deformed false joint which had developed because of a non union of the supracondylar fracture just above it, sustained in childhood which had been neglected ever since. One of the above mentioned patients, aged 24 years had undergone a French osteotomy for correction of cubitus varus, but unfortunately suffered a non union. One male patient had multiple fractures of the forearms bilaterally two years back and at the time of presentation had developed severe myositis ossificans over the right elbow. His right elbow was operated for total elbow arthroplasty after completely excising the mass of ossificans.Two females, aged 46 and 52 years had developed a serious painful fibrous ankylosis of their elbows due to clinically established rheumatoid arthritis. Three males aged 38, 48, 51 years and one female aged 62 years had sustained badly comminuted fractures of the lower end of humerus, of which the lady's fracture was compound. The preoperative range of movements of the elbows considered for replacements were nil in the patients having false, flail joints due to neglected supracondylar fractures, were very painful and restricted in cases of ankylosed rheumatoid arthritis and the elbows in comminuted fractures (bag of bones) were immobilized in a supportive cast. The lady with the compound fracture was treated with regular dressings in a supportive cast, with culture sensitivity and appropriate antibiotics and was considered for surgery only eight weeks after the infection subsided.After thorough investigations and pre anaesthetic checkups these patients were considered for total elbow replacements with a linked semiconstrained metal to metal prosthesis. The components were fixed in the bones with polymethyl methacrylate bone cement in five cases. The components used in this series were from INOR Company.

The operative technique: The patient was placed supine on the operating table with the arm in front of the chest and a sandbag below the ipsilateral shoulder. Under a tourniquet a midline postero medial skin incision was given and the ulnar nerve was protected. The triceps mechanism was elevated along with the periosteum and separated to both sides (fig.2) of the proximal ulna to expose it. The replacement of the extensor mechanism becomes easier.

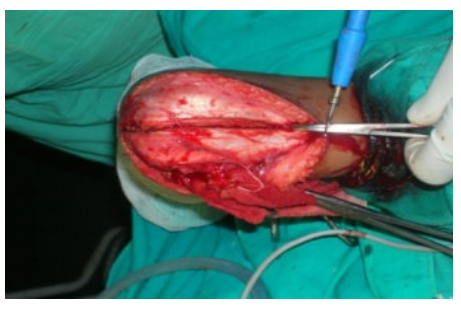

Fig 1: Midline incision in triceps after ulnar nerve

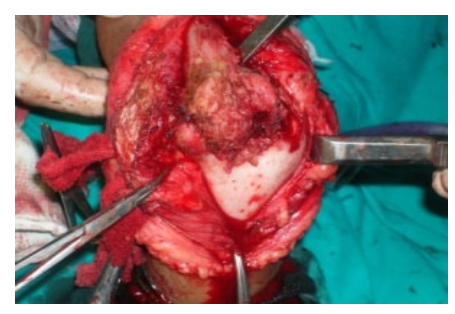

Fig 2: Triceps elevated on both sides along protecting with the periosteum Collateral ligamentsare preserved and

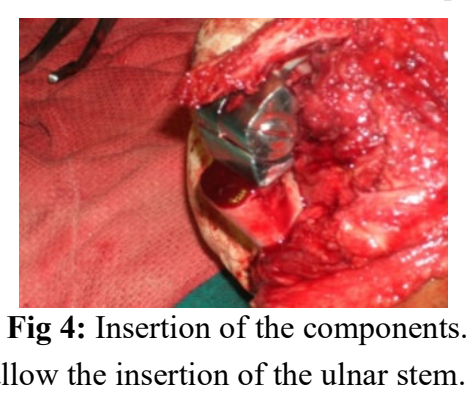

Fig 4: Insertion of the components.

Fig 3: The cut end of distal humerus.

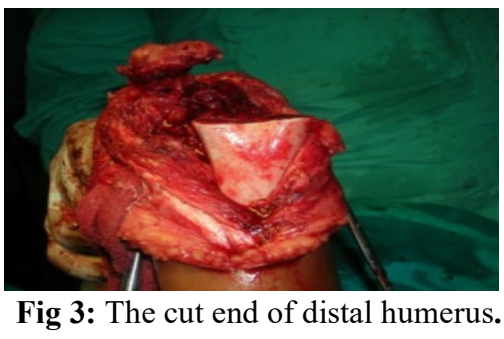

Only enough articulating surface of ulna was removed to allow the insertion of the ulnar stem. 
Research Article

The canals were reamed and then curetted with special contoured rasps. The right and left ulnar rasps correspond to ulnar stem configuration. A trial prosthesis was inserted. The prosthesis should seat exactly with the cut end of the humerus and both the components are connected with the link screw. The range of flexion and extension was examined (fig.5\& 6).

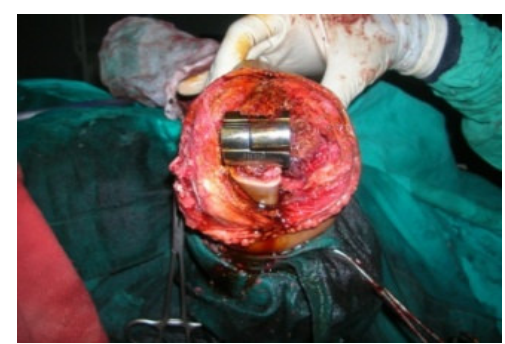

Fig 5: Full flexion after insertion of components

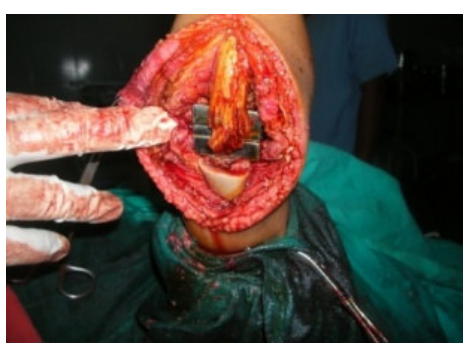

Fig 6: Full extension

Before seating the final prosthesis with poly methyl methacrylate, reassure that the radial head does not impinge on the prosthesis. The triceps tendon and the periosteum were sutured and the ulnar nerve was placed in a bed anteriorly. The tourniquet was released and hemostasis obtained. Closure was done after inserting a drain. A thick padded dressing with the elbow in a plaster splint elevated at right angles was provided. Post operatively, the extremity was elevated for 4 to 5 days with the elbow above the shoulder. The drains were removed after 48 hours, and the compressive dressing was removed on the fifth day. A light dressing was applied, and elbow flexion and extension are allowed as tolerated. An elbow immobilizer was used, and regular physiotherapy was instituted. A formal physical therapy program rarely is necessary. The patient was encouraged to avoid lifting more than 5 pounds with the involved arm for the first 3 months after surgery.
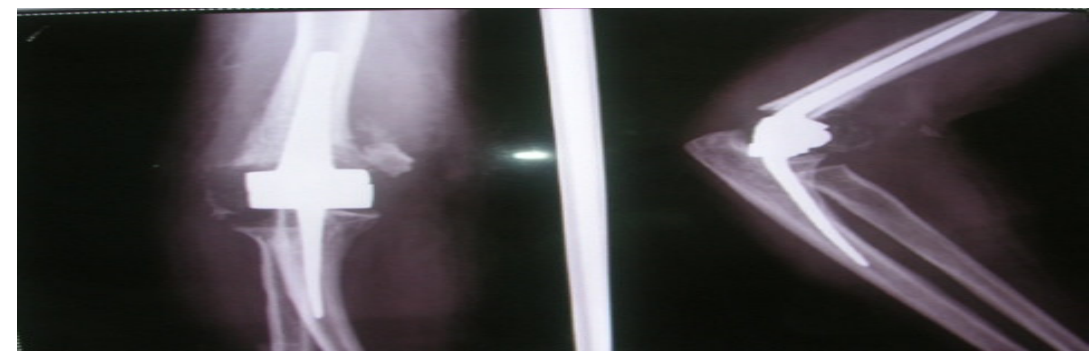

Fig 7: Post operative skiagram.

\section{Results}

Although there is no standard method of evaluating thr results of elbow implant arthroplasty, Morrey et al [13]. Inglis and Pellici [14], and Ewald [15] have evolved rating systems for evaluating the results of elbow arthroplasties. Morrey et al uses three criteria: Roentgenographic appearance, pain, and motion to determine good, fair and poor results.
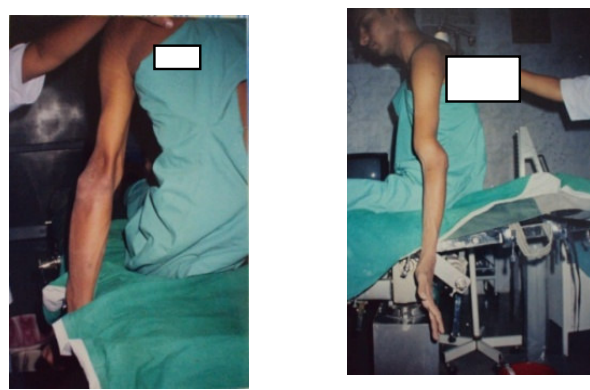

Fig 8: Before Surgery
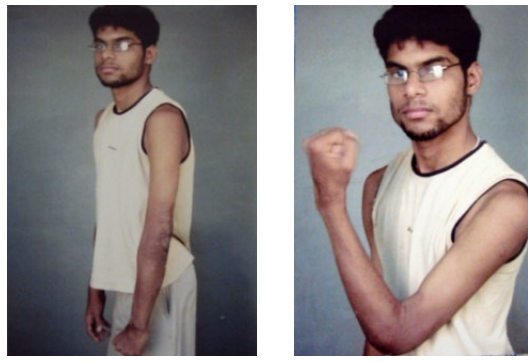

Fig 9: After Surgery

Rating system of Morrey et al [13] 


\section{Good result}

No roentgenographic change at the bone cement interface, no pain, more than 90 degrees flexion, 60 degrees of pronation and supination.

\section{Fair result}

More than $1 \mathrm{~mm}$ of widening of any bone cement prosthesis interface, mild pain, between 50 and 90 degrees of flexion, less than 40 degrees of pronation and supination.

\section{Poor result}

More than $2 \mathrm{~mm}$ of widening of any bone cement prosthesis interface, pain that significantly limits activity, less than 50 degrees of flexion and extension, less than 40 degrees of pronation and supination, revision of elbow arthroplasty.

Inglis and Pellici [14] use a scoring system with a maximum of 100 points. Pain, function, range of motion, extention contractures and pronation and supination each are assigned a numeric value. Ewald's [15] scoring system also rates pain, function, range of motion and deformity with a perfect score of 100 .
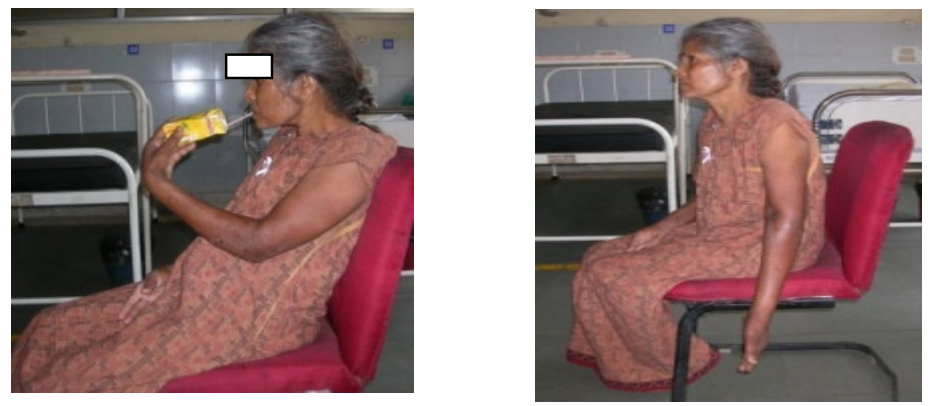

Fig 10: Limited range of flexion in a TER for a compound, comminuted fracture of distal humerus (infected).

Out of the nine patients considered, seven had no pain whereas two patients with a comminuted compound fracture of the lower end of humerus, with one patient in whom myositis was excised, complained of regular pain postoperatively. These patients developed infection post operatively. The range of movement after six weeks of surgery was more than satisfactory with a flexion of up to 130 degrees in all the cases except in one which got infected. Extension of the elbow was 180 degrees in seven cases and in one case of compound comminuted fracture and in a case of rheumatoid arthritis it fell short by 15 degrees. Supination and Pronation was more than 60 degrees in seven cases and partially restricted in two.

All the eight patients were satisfied with the range of movements achieved and the correction of deformity cosmetically. Two patients with a comminuted compound fracture suffered from pain and infection and eventually had to get the implant removed and the joint fused in the functional position. The operated elbows were stable and there was no toggling mediolaterally. According to the criteria of Morrey et al, eight cases out of nine considered were rated as good and one as poor.

\section{Discussion}

The Coonrad-Morrey prosthesis, a linked semiconstrained implant with a humeral component, porouscoated distally and an anterior flange is most commonly used currently. It increases the rotational stability of the implant. The benefit of an anterior flange has been investigated for other implants also [16]. Joint stability even with bone and ligamentous insufficiency is ensured with linked implants. A major advantage with linked implants is the ease of soft tissue dissection even in the presence of preoperative deformity and stiffness. Unlinked prosthesis is prone to dislocations and entails extensive soft tissue dissection. Their designs often demand the presence of humeral condyles and ulnar notch for their component fixation, whereas the linked prosthesis may also be used in the presence of bone loss.
According to Little et al.[17] the revision rates have been similar for linked and unlinked implants, although radiographic loosening seems to be higher with unlinked implants, especially the humeral component. Levy et al. reported a higher rate of revision for unlinked compared to linked implants. [18]

Depending on the rigidity of the fixation of the humeral component to the ulnar component, the implant arthroplasties are designated as constrained, semiconstrained, and unconstrained [19]. In general, the constrained prostheses rarely are used because of their tendency to loosen and break. In salvage situations in which bone loss has been extensive, prosthesis with a firmly connected humero ulnar articulation might be 
appropriate. Totally constrained, metal-to-metal prostheses include the Stanmore, Dee, McKee, GSB I (Gschwend, Scheier, and Bähler), and Mazas designs. The totally constrained elbow arthroplasties generally have a metal-to-metal hinge with polymethylmethacrylate bone cement fixation.

The semiconstrained prostheses are two- or three-part prostheses that have a metal-to-high-densitypolyethylene articulation, which may be connected with a locking pin or with a snap-fit device. The semiconstrained hinged prostheses have built-in valgus and varus laxity to provide for dissipation of forces. The GSB III, HSS-Osteonics, and the Coonrad-Morrey prostheses are semiconstrained [19].

The unconstrained prostheses usually are two-part devices consisting of metal articulating with high-density polyethylene. They usually do not have a snap-fit, link, or pin connection. Some designs consist of a resurfacing device, and some have stems for the humeral component. The unconstrained implant arthroplasties include the capitellocondylar (Ewald), London, Kudo, Ishizuki, Lowe-Miller, Wadsworth, and Souter designs. Most of these prostheses are unlinked in an attempt to anatomically duplicate the articular surfaces of the elbow.

They restore the joint's anterior offset from the humerus and have a single center of rotation. All resurfacing or unconstrained prostheses require normal intact ligaments and anterior capsule, as well as appropriate static alignment. The resurfacing arthroplasties can be divided further into two groups: those that maintain the normal relationship between the humerus and the forearm in the frontal plane and those that realign the medullary canal of the ulna with the medullary canal of the humerus. If bone loss or capsuloligamentous destruction is extensive, an unconstrained prosthesis generally cannot be used.

Unlinked implants are attractive for patients with relatively well preserved bone stock and ligaments, but many favor linked implants, since they prevent instability and allow replacement for a wider spectrum of indications. Inflammatory arthropathies such as rheumatoid arthritis represent the classic indication for elbow arthroplasty [20]. Indications have been expanded to include posttraumatic osteoarthritis, acute distal humerus fractures, distal humerus nonunions and reconstruction after tumor resection [21]. Elbow arthroplasty is very successful in terms of pain relief, motion and function. However, its complication rate remains higher than arthroplasty of other joints. The overall success rate is best for patients with inflammatory arthritis and elderly patients with acute distal humerus fractures, worse for patients with posttraumatic osteoarthritis [22]. The most common complications of elbow arthroplasty include infection, loosening, wear, triceps weakness and ulnar neuropathy [23]. When revision surgery becomes necessary, bone augmentation techniques provide a reasonable outcome.

\section{Conclusion}

Selection of the type of prosthesis implant depends to a great extent on the state of capsuloligamentous structures about the elbow and the integrity of musculature, as well as the amount of bone remaining at the elbow joint. It is important to remember that the goals of reconstructive elbow surgery are to restore function through the relief of pain and the restoration of motion and stability.

\section{Funding: Nil}

\section{Conflict of interest: Nil}

\section{Permission from IRB: Yes}

\section{References}

1. Riseborough EJ, Radin EL. Intercomdylar T fractures of the humerus in the adult: a comparison of operative and non-operative treatment in twenty-nine cases. $\mathrm{J}$ Bone Joint Surg Am 1969;51(1):130-41.

2. Brown RF, Morgan RG. Intercondylar T-shaped fractures of the humerus: results in ten cases treated by early mobilisation. J Bone Joint Surg $\mathrm{Br}$ 1971; 53(3):425-8.

3. Gofton WT, Macdermid JC, Patterson SD, et al. Functional outcome of AO type $\mathrm{C}$ distal humeral fractures. J Hand Surg [Am] 2003;28(2):294-308.

4. Korner J, Lill H, Muller LP, et al. Distal humerus fractures in elderly patients: results after open reduction and internal fixation. Osteoporos Int(Suppl 2):S73-9.

5. Kundel K, Braun W, Wieberneit J, et al. Intraarticular distal humerus fractures: factors affecting functional outcome. Clin Orthop Relat Res 1996;332:200-8.

6. Mckee MD, Jupiter JB. A contemporary approach to the management of complex fractures of the distal humerus and their sequelae. Hand Clin 1994;10(3):47994.

7. Pajarinen J, Bjoerkenheim JM. Operative treatment of type $\mathrm{C}$ intercondylar fractures of the distal humerus: results after a mean follow up of 2 years in A series of 18 patients. J Shoulder Elbow Surg 2002;11(1):48-52. 
8. Ring D, Jupiter JB. Complex fractures of the distal humerus and their complications. J Shoulder Elboe Surg 1999; 8(1):85-97.

9. Robinson CM, Hill RM, Jacobs N, et al. Adult distal humeral metaphyseal fractures: epidemiology and results of treatment. J Orthop Trauma 2003;17(1):38-47.

10. Sanders RA, Raney EM, Pipkin S. Operative treatment of bicondylar intraarticular fractures of the distal humerus. Orthopaedics 1992;15(2):159-63.

11. Aitken GK, Rorabeck CH. Distal humeral fractures in the adult. Clin Orthop Relat Res 1986;207:191-7.

12. Fevang BT, Lie SA, Havelin LI, Skredderstuen A, Furnes O. Results after 562 total elbow replacements: a report from the Norwegian Arthroplasty Register. J Shoulder Elbow Surg. 2009;18(3 ):449-56.[PubMed]

13. B. F. Morrey, L. J. Askew, K. N. An, and E. Y. Chao, "A biomechanical study of normal functional elbow motion," Journal of Bone and Joint Surgery A, vol. 63, no. 6, pp. 872-877, 1981.

14. Inglis A.E., Pellicci P.M. Total elbow replacement J Bone Joint Surg [Am] 1980 ; 62A : 1252-1258.

15. Ewald FC, Scheinberg RD, Poss R, Thomas WH, Scott RD, Sledge CB. Capitellocondylar total elbow arthroplasty. J Bone Joint Surg Am. 1980 Dec;62 (8):1259-63.

16. Quenneville CE, Austman RL, King GJ, Johnson JA, Dunning CE. Role of an anterior flange on cortical strains through the distal humerus after total elbow arthroplasty with a latitude implant. J Hand Surg Am. 2008;33 (6): 927-31.
17. Little CP, Carr AJ, Graham AJ. Total elbow arthroplasty: a systematic review of the literature in the english language until the end of 2003. J Bone Joint Surg Br. 2005;87(4):437-T44.

18. Levy JC, Loeb M, Chuinard C, Adams RA, Morrey BF. Effectiveness of revision following linked versus unlinked total elbow arthroplasty. J Shoulder Elbow Surg. 2009;18(3):457-62.

19. Joaquin Sanchez-Sotelo .Total Elbow Arthroplasty. Open Orthop J. 2011; 5: 115-123. Published online Mar 16, 2011. doi: 10.2174/1874325001105010115.

20. Khan HA. Primary total elbow arthroplasty. Indian J Orthop. 2014 Sep;48(5):536. doi: 10.4103/0019-5413. 139891.

21. Gay DM, Lyman S, Do H, Hotchkiss RN, Marx RG, Daluiski A. Indications and reoperation rates for total elbow arthroplasty: an analysis of trends in New York State. J Bone Joint Surg Am. 2012 Jan 18;94(2): 110-7. doi: 10.2106/JBJS.J.01128.

22. Mansat $\mathrm{P}$, Bonnevialle $\mathrm{N}$, Rongières $\mathrm{M}$, Bonnevialle P; Bone, Joint Trauma Study Group. The role of total elbow arthroplasty in traumatology. Orthop Traumatol Surg Res. 2014 Oct;100(6 Suppl):S293-8. doi: 10.1016/j.otsr.2014.06.008. Epub 2014 Aug 20.

23. Toor AS, Jiang JJ, Shi LL, Koh JL. Comparison of perioperative complications after total elbow arthroplasty in patients with and without diabetes. J Shoulder Elbow Surg. 2014 Nov;23(11): 1599-606. doi: 10.1016/j.jse.2014.06.045. Epub 2014 Sep 9

\section{How to cite this article?}

Saran R, Saxena A. Evaluation of results of Total Elbow Arthroplasty with a Linked Semiconstrained Prosthesis. Int J Med Res Rev 2014;2(6):538- 543. doi:10.17511/ijmrr.2014.i06.06 